\title{
Textos eficaces, alumnos felices: técnicas y reflexiones para mejorar la competencia discursiva de los aprendientes de E/LE
}

\author{
JULIÁN MuÑOZ PÉREZ
}

Universidad Complutense de Madrid/ EOI de Madrid-Embajadores

julian.munoz@ucm.es

\begin{abstract}
Resumen: El objetivo de este trabajo es proporcionar a los profesores de E/LE una serie de herramientas para que sus alumnos puedan ejercitar su competencia discursiva con los textos que reciben y producen. Estas técnicas responden a un enfoque didáctico basado en los textos y en los géneros discursivos, sensible a las diferencias que pueden dificultar la comprensión y la expresión del alumno no nativo, ya sean de orden intercultural (en el plano retórico) o lingüístico (en el manejo del repertorio de los dispositivos léxicogramaticales que articulan los textos). Presentaremos una justificación teórica de nuestro enfoque y cuatro propuestas de actividades para los niveles A2-C2 del Marco Común Europeo de Referencia (MCER), centradas en la estructura de los géneros discursivos, la cohesión léxica y la coherencia textual.
\end{abstract}

Palabras clave: competencia discursiva, coherencia, cohesión, enfoque textual.

\section{Effective texts, happy students: some techniques and reflections to improve discourse competence in Spanish L2 learners}

\begin{abstract}
The main purpose of this article is to provide Spanish teachers with a series of tools which can help students develop their discourse competence when processing the texts they receive and produce. These techniques rely on a text- and genre-based approach which takes account of the differences that can make non-native learners' comprehension and expression difficult in an L2. In particular, these difficulties may respond to intercultural or linguistic divergences, both in terms of rhetorical patterns and lexicogrammatical devices assembling a text. We will offer a theoretical framework of our approach and present four activities focused on genre structure, lexical cohesion and textual coherence from an $\mathrm{A} 2$ to a $\mathrm{C} 2$ level as defined by the CEFR.
\end{abstract}

Key words: discourse competence, coherence, cohesion, text-based approach.

\section{Fundamentos epistemológicos, neuropsicológicos y praxeológicos}

Señala McGrath (2016:92) que "todas las muestras de lengua a las que se expone a los alumnos y que se espera que produzcan deberían ser realistas, es decir, potencialmente utilizables y útiles". Sin embargo, los profesores de E/LE, después de dedicar varias sesiones al análisis y reflexión sobre el sistema de la lengua y su uso en las diferentes destrezas, nos enfrentamos no pocas veces a producciones de nuestros alumnos en las que echamos en falta precisamente ese realismo o naturalidad. Más allá de los errores y faltas que puedan cometer, podemos tener la impresión de que nuestros alumnos no están comunicando todo lo que podrían (informatividad), de que su expresión resulta inconexa (cohesión) o carente de cierta lógica (coherencia) o de que no responden a los patrones empleados por los hablantes de la lengua meta (aceptabilidad). 
Por otra parte, quienes se ocupan de la enseñanza de una L1 han llegado a la conclusión de que el incremento en la cantidad y profundización de los conceptos lingüísticos programados en los currículos de lengua(s) materna(s) -incluidos los provenientes de la lingüística del texto- no se ha traducido en una mejora a medio o largo plazo de las destrezas productivas - ni de las comprensivas- de los alumnos (Ferrer y Rodríguez 2010:97). El desarrollo de la competencia discursiva no queda, pues, satisfecho con la aglomeración de nuevos contenidos conceptuales sobre otros más tradicionales, sino que requiere de una atenta reflexión en los tres órdenes fundamentales de la didáctica -el epistemológico, el neuropsicológico y el propiamente praxeológico (Álvarez Angulo 2005:49)- si se quiere que los alumnos de L1 y de L2 mejoren sus capacidades de procesamiento y elaboración de secuencias textuales y de géneros discursivos realistas.

Con este panorama en mente, nuestro objetivo consiste en presentar algunas técnicas didácticas sustentadas en un marco de actuación que contempla dos variables. Por un lado, el sistema de creencias en que se apoya toda metodología, y que es fruto de la reflexión académica y profesional acumulada a lo largo del tiempo. Por otro, las pruebas empíricas - de orden neurolingüístico, psicolingüístico y pedagógico- que respaldan o refutan las ideas anteriores. Las exponemos seguidamente, de un modo muy sucinto, para que el lector pueda comprender mejor nuestra propuesta y analizarla críticamente.

Como marco conceptual, partimos de la premisa sustancial de que la lengua se manifiesta esencialmente como texto y discurso (Fuentes Rodríguez 2000:12; Moreno Cabrera 2002:759; Bronckart 2007:74), y no como sintagmas, oraciones o colocaciones léxicas azarosas. Sin desatender los avances importantes experimentados por los estudios gramaticales y lexicológicos, entendemos que las unidades lingüísticas cobran su verdadero sentido en el marco de los textos; esto es, de secuencias de lengua efectivamente enunciadas (Hymes 1971[1995:41]). Por su parte, los textos y los discursos, a pesar de gozar de un alto margen de creatividad y variedad en diacronía (Bronckart 2007:79), responden en sincronía a ciertos patrones que los hacen predecibles y manejables (Serrano 2006:14; Cuenca 2008:13; Duque 2016:5), de modo que son susceptibles de ser analizados, descritos y, en consecuencia, enseñados.

Tras haber analizado varios modelos lingüísticos y pedagógicos de competencia comunicativa propuestos desde los años 80 (Muñoz Pérez 2017:101-124), estamos convencidos de que la subcompetencia discursivo-textual es el verdadero eje de aquella (Celce-Murcia, Dörnyei y Thurrell 1995:10; Alonso Belmonte 2004:556) puesto que correlaciona armónicamente los componentes lingüístico, pragmático, sociocultural y estratégico en una práctica discursiva propia de la comunidad meta. En consonancia, los aprendientes adquieren mejor la lengua cuando actúan como analistas del discurso (Riggenbach 1999:36-47; Wennerstrom 2003:3), esto es, procesando y elaborando muestras de lengua auténticas y contextualizadas.

En cuanto a los datos empíricos, los estudios apoyados en electroencefalogramas (van Berkum 2012:605) y resonancias magnéticas cerebrales (Hasson y Small 2008:86) revelan que la diferencia entre procesamiento de la oración y del discurso es de orden cuantitativo, y no tanto cualitativo: afecta a las mismas áreas cerebrales del hemisferio izquierdo, pero en redes más extensas y con señales eléctricas más intensas. De ahí que dichas conexiones requieran ser estimuladas y reforzadas en el proceso de aprendizaje mediante el trabajo con textos completos o con fragmentos amplios, por lo que habría que replantearse la efectividad de las prácticas basadas en enunciados o frases aislados. 
No obstante lo anterior, los materiales de E/LE generalmente se articulan siguiendo un currículo centrado en la morfosintaxis oracional -e incidentalmente en la conectividad (Muñoz Pérez 2017:531)- y recurriendo a secuencias y ejercicios de la era estructural cuya eficacia psicolingüística o pedagógica -como ocurre con la secuencia PPP- no ha sido aún empíricamente demostrada (Andon y Wingate 2013:190; Masuhara 2016:29). Además, la gran mayoría del input lo constituyen textos modelados por el reducido equipo de autores de los materiales (Muñoz Pérez 2017:457), lo cual puede ser contraproducente para el aprendizaje, ya que se alejan de las prácticas reales de la comunidad meta e impiden la exposición a un input significativo y rico (Burns 2012:147). Tampoco contienen apenas propuestas didácticas sobre cohesión léxica, secuencias textuales, desarrollo temático o coherencia, sino que parecen reducir el nivel discursivo-textual al uso de los marcadores y organizadores del discurso (Muñoz Pérez 2017:396), ignorando, por tanto, buena parte de los demás dispositivos discursivos y textuales a los que recurren los nativos en sus prácticas comunicativas cotidianas.

Con todas estas claves en mente, procedemos a describir las tareas y las actividades que hemos diseñado y pilotado con alumnos de español jóvenes y adultos de varias nacionalidades que estudian en un centro público en situación de inmersión (EOI), con las que buscamos estimular ulteriores desarrollos no solo por quien redacta este artículo, sino también por quienes lo leen y trabajan con otros perfiles y en contextos diferentes.

\section{Tareas holísticas: análisis contrastivo e intercultural de modelos textuales}

Los modelos de enseñanza de la lengua basada en el texto, pese a algunas divergencias superficiales, suelen coincidir en arrancar la secuencia didáctica a partir de la deconstrucción de un modelo textual y de la situación de enunciación en que se enmarca (Feez y Joyce 1998:28; Tomlinson 2013:24). En esta fase se procura que el alumno observe el texto como artefacto desde una perspectiva global y que entienda cómo se organiza comunicativamente tanto desde un punto de vista textual como paratextual.

Es importante, pues, que los aprendientes tengan la oportunidad de enfrentarse a los textos no solo para detectar muestras de determinados recursos formales (texto como pretexto, según Maley y Prowse 2013:166), sino para entender que aquellos responden globalmente a una función comunicativa y a una configuración retórica convencionalizada por las expectativas de la comunidad de hablantes. Igualmente, debemos alertarles sobre las posibles divergencias en la denominación, configuración y función de los géneros discursivos entre la cultura propia y la meta. Obviar esto puede conducir a que los alumnos transfieran patrones inapropiados de su lengua que acaben afectando a la informatividad, la coherencia o la aceptabilidad de sus producciones.

En nuestro caso, proponemos que los alumnos, en función de su nivel general de competencia, confeccionen unas fichas con información sobre los diferentes géneros textuales a que se van enfrentando a lo largo del curso, de modo que no solo se desarrolle un trabajo ocasional sobre cada texto, sino que se les dote a medio y largo plazo de herramientas que los conviertan en analistas del discurso autónomos. Estas fichas, que van de lo más inmediato a lo más inferencial, se basan en los conceptos de superestructura (Martín Peris 2008:537), macroestructura (Martín Peris 2008:341) y situación de comunicación (Feez y Joyce 1998:28), a los que nos referiremos seguidamente. Cada uno de estos fenómenos se trabaja en dos fases. 
Así, por ejemplo, en los niveles A2 y B1 del MCER podemos desarrollar algunas tareas orientadas al análisis de la superestructura (es decir, de los rasgos superficiales más evidentes). Siguiendo a Tomlinson (2013:24), les proponemos en un primer momento a los alumnos que respondan espontáneamente a un estímulo (input) movilizando todos los recursos de que disponen en ese momento (output). Este educto inicial les hará tomar conciencia de sus necesidades comunicativas y motivará toda la secuencia. Una vez hecho esto, se les proporciona un modelo de texto que responda al mismo input inicial. Después de haber trabajado en su comprensión, y con ayuda del inventario de "Géneros discursivos y productos textuales" del Plan Curricular del Instituto Cervantes (2006) o cualquier otra herramienta similar, deben responder a las preguntas 1 a 3. En la Fase II contrastarán su propio educto con el modelo de texto trabajado, e incluso puede optarse por hacer el contraste con un modelo propio de la cultura del aprendiente previamente seleccionado (lo cual funcionará mejor en contextos con una L1 común).

(1) Modelo de ficha para los niveles A2 y B1

FASE I. CATEGORIZACIÓN

1. Observa el texto que has leído. ¿Qué tipo de texto es?

2. Divide el texto en partes, según los diferentes movimientos.

3. ¿Cuál es la función de cada una de esas partes? ¿Qué nombres les pondrías?

FASE II. CONTRASTE

4. Observa el texto que habías escrito previamente. ¿Tiene las mismas partes que el modelo?

5. ¿Existe este tipo de textos en tu cultura? ¿Cómo se llaman? ¿Hay diferencias entre la organización del modelo en español y un modelo del mismo tipo de texto en tu lengua?

En el nivel B2 la tarea se centraría en el estudio de la macroestructura (esto es, la organización semántica e informativa) de modo que se analizarían, por un lado, las secuencias textuales y su organización (Martín Peris 2008:524), y, por otro, se propondrían tareas para diferenciar formal y funcionalmente géneros afines (por ejemplo, el chiste, la anécdota, el monólogo de humor o el meme):

(2) Modelo de ficha para el nivel B2

FASE III. SECUENCIACIÓN

6. ¿Qué tipos de secuencias textuales aparecen en el texto: narración, descripción, diálogo...?

7. ¿Cuál es la que sirve para articular el texto? ¿Cómo se relacionan las demás con ella?

8. Realiza un esquema de cómo se organiza secuencialmente el contenido del texto.

FASE IV. RELACIÓN

9. ¿Conoces otros tipos de textos que tengan una estructura textual similar? ¿En qué se parecen a este? ¿En qué se diferencian?

Por último, en los niveles C1 y C2 lo que interesa es que los alumnos sean capaces de vincular el texto con la situación de comunicación; esto es, que se conciencien de la influencia que ejercen las condicionantes de campo (tema del discurso), tenor (los participantes, sus roles y actitudes) y modo (canal de transmisión) sobre las formas y las funciones del texto. También les puede resultar útil retener algunas fórmulas retóricas más o menos fijas en cada género, reutilizables en sus producciones ulteriores. 
(3) Modelo de ficha para los niveles $\mathrm{C} 1$ y $\mathrm{C} 2$

FASE V. SITUACIÓN

10. ¿Cuál es el tema del texto? ¿En qué otros tipos de textos se podría hablar del mismo tema? ¿De qué otros temas se podría hablar en este tipo de texto?

11. ¿Cómo se manifiesta la presencia del emisor en el texto? ¿Y la del receptor del mensaje? ¿Qué tipo de relación mantienen? ¿Cuáles son sus actitudes: ironía, distancia, afecto...?

12. ¿Cuál es el canal del texto? ¿Qué recursos lo evidencian?

FASE VI. RETÓRICA

13. ¿Qué expresiones fijas suelen usarse en tu lengua en este tipo de texto?

14. Selecciona entre seis y diez expresiones de este texto que crees que pueden serte útiles.

En definitiva, se propone un trabajo acumulativo y progresivo que permite que un mismo género discursivo sea recuperado en los sucesivos niveles, poniendo en cada ocasión el centro de interés en un aspecto discursivo diferente. Una vez que los alumnos han trabajado el texto de esta manera, los modelos didácticos de enseñanza basada en textos invitan a que se aproximen a determinados fenómenos textuales, lingüísticos, pragmáticos o socioculturales que requieren de un tratamiento pormenorizado; si se prefiere, como tareas de atención a la forma. A ello nos dedicamos seguidamente.

\section{Tareas de atención a la forma: cohesión léxica y coherencia}

Sin duda, son muchos los aspectos discursivo-textuales que pueden ser objeto de tratamiento en esta fase de reflexión sobre la forma. Nos limitaremos aquí a sugerir dos propuestas para trabajar la cohesión léxica y otra relacionada con la coherencia textual, fenómenos poco tratados en los materiales de E/LE (Muñoz Pérez 2017).

\subsection{Las etiquetas del discurso como estrategia tematizadora}

Según Duque (2016:66) las etiquetas de discurso son unidades léxicas (generalmente sustantivos) que «simplifican la estructura textual al resumir o encapsular el discurso precedente y facilitan, así, su conexión con otros segmentos textuales». Además de esta caracterización textual, morfológicamente suelen ser derivados deverbales cuya sintaxis puede regir determinadas preposiciones, y que además reclaman ciertas colocaciones. Precisamente esta capacidad de activar conjuntamente todos los niveles de la lengua nos parece altamente recomendable trabajarlas en el aula.

Podemos proponer a nuestros alumnos, a partir del nivel B2, que lean una serie de fragmentos periodísticos auténticos como los de los ejemplos (4), (5) y (6):

(4) Alemania: cuatro presos se escapan de cárcel por agujero en muro

[ ] a través de un agujero que los convictos cavaron en un muro del sistema de ventilación. (http://larepublica.pe, 28/12/2017)

(5) La mancha de petróleo del 'Sanchi' triplica su extensión y amenaza la ruta migratoria de ballenas

La Administración Oceánica Estatal china ha informado de tres machas distintas que han pasado en cuestión de cuatro días de 101 a 332 kilómetros cuadrados en total. 
[ ] a varios mamíferos marinos que pasan por la región en sus migraciones. (http://www.antena3.com, 22/01/2018)

(6) Los amantes de la cerveza están de enhorabuena: una cervecera austriaca ha creado la primera piscina de cerveza del mundo. La responsable de hacer realidad el sueño de muchos ha sido la compañía Starkenberger, que ha instalado un complejo muy especial en un castillo ubicado en Tarrenz, en el estado de Tirol (Austria), a partir del diseño del artista Wernfried Poschusta.

[ ] de la creencia de que esta bebida tiene propiedades medicinales para la piel, por este motivo recomiendan no ducharse de inmediato, tras disfrutar de una de sus sesiones, para propiciar que el líquido amarillo haga sus efectos en el cuerpo. (http://www.laverdad.es, 08/08/2017)

Lo primero que pedimos a los alumnos es que completen las líneas de cada texto con un sustantivo determinado que resuma el contenido de lo hasta entonces mencionado. Así, en (4) podrían servir sintagmas nominales como la huida o la fuga; en (5) el vertido o el derrame; y en (6) la idea o esta iniciativa. Tras discutir diferentes opciones sobre los sustantivos y los determinantes que cabrían en esa posición y su función tematizadora, se les pide que continúen el fragmento con una forma verbal que sustituya el espacio marcado entre paréntesis. Algunas soluciones posibles serían se produjo y tuvo lugar para (4), afecta o puede dañar en (5), y surge, parte, se basa en o proviene para (6).

A diferencia de los ejercicios de huecos tradicionales (Alderson, Clapham y Wall 1995:56), no hay aquí una única respuesta correcta, sino que cada propuesta destaca distintos aspectos significativos que pueden negociarse, lo cual es más enriquecedor. Además, este ejercicio puede complementar bien a otros más tradicionales en los que se rellenan tablas con derivados a partir de una forma primitiva o en que se listan las posibles colocaciones de una lexía, pero que al carecer de contexto -mejor dicho, de sentidoprovocan que el aprendizaje sea menos consistente a medio y largo plazo.

\subsection{Las redes léxicas y su función cohesiva}

Otro de los mecanismos que proporcionan cohesión a un texto son las redes léxicas: cadenas de elementos léxicos que contribuyen a mantener la referencia, a la vez que matizan y precisan el significado. Ayudar a los alumnos a detectar estas redes y su funcionamiento les puede resultar muy útil para mejorar su expresión, además de para organizar el léxico que adquieren dentro y fuera del aula de un modo más eficiente.

Esta propuesta se dirige a alumnos de nivel $\mathrm{C}$ a los que se les pide que marquen en un texto ya trabajado aquellos grupos de palabras que mantienen algún tipo de relación semántica. Por ejemplo, en la entrada de la Wikipedia relativa al dragón de Komodo aparecen los siguientes verbos para explicar su alimentación: se alimentan, sean devoradas, tragándoselos, sin consumir, comen, ingerir, sin digerir o regurgita.

Una vez que se han detectado esos elementos léxicos, y que se ha fijado cuál es el archilexema o término general que los vincula (comer), el reto consiste en completar la ficha de la imagen 1 con dos condiciones: (i) que ninguna palabra de la parte superior (relaciones léxicas) repita la letra inicial; y (ii) que solo se puede trabajar la fila inferior (derivación) si se ha conseguido completar con éxito la tarea anterior. Estas pautas, que 
ayudan a que el caudal de vocabulario que manejan y descartan los alumnos sea más rico, pueden flexibilizarse según el grado de dificultad del texto o del campo léxico (por ejemplo, pueden repetir solo una letra o trabajar la ficha en varias sesiones).

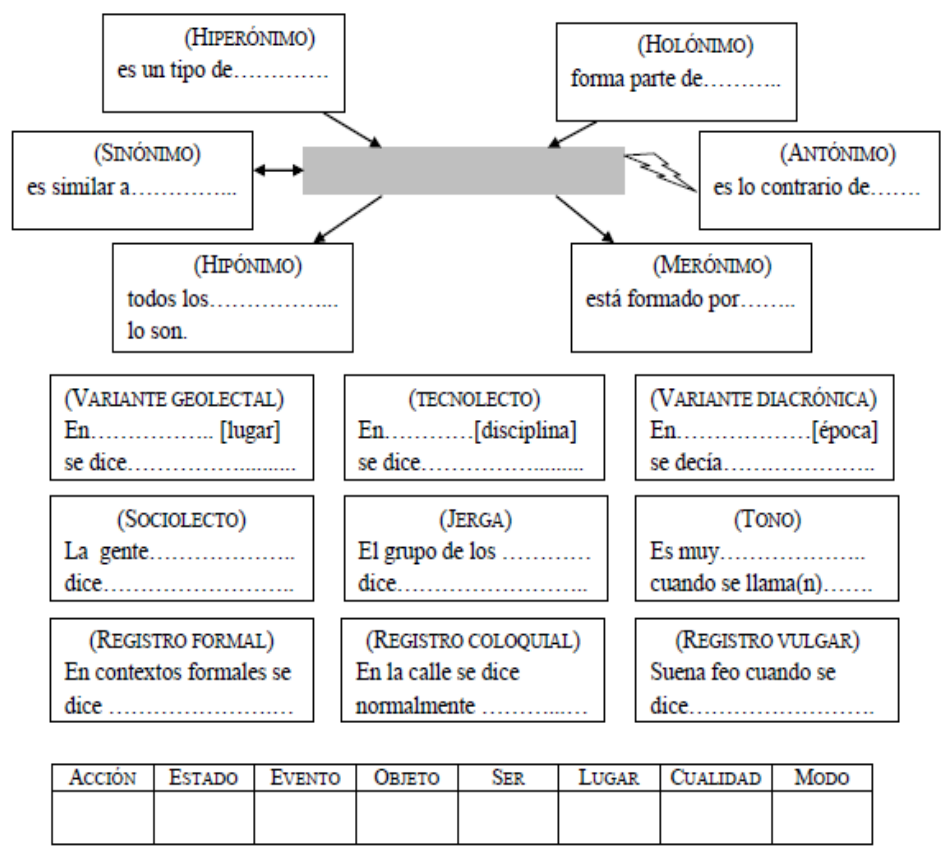

Imagen 1. Ficha para trabajar las relaciones léxicas.

Los elementos léxicos descubiertos en el texto, junto a otros que pueden buscar en diferentes tipos de fuentes (como diccionarios o corpus), permitirán a los alumnos tomar conciencia de la especialización que adquieren, en nuestro caso, determinados verbos en relación con comer (consumir [hiperónimo], sustentarse [holónimo], ingerir [sinónimo], ayunar [antónimo], merendar [hipónimo], tragar [merónimo], buyonear [dialecto lunfardo], nutrirse [tecnolecto], yantar [español medieval], papear [juvenil], jamar [jerga gitana], degustar [formal], zampar [coloquial], llenarse el buche [vulgar]), así como de las formas léxicas no verbales emparentadas (comida [evento], comensal [persona], comedero [objeto], comedor [lugar], comestible [cualidad]).

Este ejercicio supone un verdadero reto cognitivo (incluso para los docentes) que motiva a los alumnos con un alto nivel de competencia. Pero lo esencial es que, con independencia de lograr completar la tabla o no, les ayude a observar los textos con una mirada diferente y a revisar sus producciones para aspectualizar y matizar los referentes y los significados que lo cohesionan. Lo ideal es que, una vez asimilada la dinámica, propongan ellos sus propios textos para analizar o los campos léxicos que les interesan.

\subsection{Coherencia textual y configuración retórica}

La construcción de los textos responde a un planteamiento lógico de presentación de su contenido informativo: causa-consecuencia, problema-solución, tesis-antítesis-síntesis, etc. (McCarthy y Carter 1994:55). En este sentido, tener bien planificada una estrategia retórica puede resultar mucho más útil que saturar el texto con marcadores $\mathrm{y}$ organizadores discursivos que dificultan la comprensión y resultan redundantes 
(Thornbury 2005:34). Además, es un criterio de evaluación fundamental tanto en los exámenes oficiales de certificación del idioma (DELE, EOI) como en las tareas que proponen los manuales de E/LE (Muñoz Pérez 2017:527), y, sin embargo, no suele recogerse explícitamente entre las propuestas didácticas de dichos materiales.

Un ejemplo de texto construido conforme a un plan retórico de antítesis-síntesis es la homilía «Fidelidad a Dios y a la Tierra» (Pagola 2011:161). Sin ser polémico desde el punto de vista del contenido (ecología y cristianismo), y dado que el MCER cita explícitamente el sermón como un género que puede interesar a los aprendientes, especialmente en situación de inmersión (por practicar alguna religión cuyos ritos se ofician en español, por tener que asistir a ceremonias como bodas o funerales, etc.), nos parece que ilustra muy bien la estrategia de persuasión y adhesión que persigue su autor de un modo indirecto pero sostenido (típica de los discursos religiosos, filosóficos e ideológicos que conviene trabajar en los niveles C). En todo caso, pueden buscarse alternativas a este texto si se considera culturalmente comprometido, pero nos parece una oportunidad de enriquecer el corpus de textos al que exponemos a los aprendientes (vid. 2) y de trabajar temas que pueden suscitar reacciones entre ellos -incluso de crítica o de rechazo-, lo cual favorece siempre el aprendizaje frente a textos afectivamente planos (Tomlinson 2013:12) e irrelevantes (Andon y Wingate 2013:186).

Tras leer el texto, los alumnos de nivel B2 deben señalar en el mismo las marcas lingüísticas mediante las que el autor confronta las diferentes actitudes de los dos grupos en liza: el de los cristianos "ascetas" despreocupados por las cuestiones mundanas, y el de los ecologistas no creyentes. Hallamos así los siguientes ejemplos:

(7) «los que oran no hacen la revolución, y los que hacen la revolución no oran».

(8) hay quienes buscan a Dios sin preocuparse de buscar un mundo mejor y más humano. Y hay quienes pretenden construir una tierra nueva sin Dios.

(9) Unos buscan a Dios sin mundo. Otros buscan el mundo sin Dios. Unos creen poder ser fieles a Dios sin preocuparse de la tierra. Otros creen poder ser fieles a la tierra sin abrirse a Dios.

Como puede verse, los antagonistas se contraponen mediante estructuras del tipo "los que A no B, y los que B no A", "hay quienes C $\sin \mathrm{D}$. Y hay quienes $\mathrm{D} \sin \mathrm{C}$ " que hacen patente su punto de vista complementario, y cuyas diferencias se desarrollan en más detalle con la secuencia progresiva y paralela "Unos $X^{1}$. Otros $X^{2}$. Unos $Z^{1}$. Otros $Z^{2}$ ". Pero es precisamente esa complementariedad (aparentemente irreductible) la que permite sintetizar, reconciliándolas, ambas posturas en el párrafo final:

(10) Quien se abre intensamente a Dios, ama intensamente la tierra. Quien se encuentra con el Dios de Jesucristo, siente con más fuerza la injusticia, el desamparo y la autodestrucción de los hombres.

Es decir, pese a la aparente divergencia de ambas cosmovisiones, se concluye afirmando la existencia de un punto de encuentro ("Quien E, F. Quien F, E”); conclusión a la que se llega gracias a repeticiones anafóricas que distribuyen la información (lo temático se convierte en remático, y viceversa) sin usar dispositivos macrosintácticos explícitos.

A partir de este modelo, podemos pedir a los alumnos que, individualmente o en grupos, seleccionen una pareja de aparentes antagonistas (perros y gatos, mayores y jóvenes, hombres y mujeres, conservadores y progresistas, tauro y leo, etc.) y redacten un texto 
usando esas mismas estructuras a modo de esqueleto, pero sin desvelar de quiénes están hablando. Después los textos se compartirán para que los compañeros puedan adivinar de qué par se trata y, si procede, organizar algún tipo de investigación o debate.

\section{Conclusión}

A lo largo de este trabajo hemos ofrecido algunas reflexiones y estrategias para mejorar la competencia discursiva de los aprendientes de E/LE. Se trata de actividades sencillas y que no precisan de muchos medios, pero fundamentadas desde un punto de vista teórico y empírico. Esperamos que a partir de ellas y de las lecturas que ofrecemos en la bibliografía, los profesores puedan seguir aportando nuevas perspectivas de análisis de la lengua y de práctica en el aula que favorezcan la autonomía de nuestros alumnos, quienes por fuerza habrán de enfrentarse a discursos que no han sido tratados en clase. Con estos instrumentos, creemos que ese reto les resultará más viable y satisfactorio.

\section{Bibliografía}

Alderson, J. Charles, Clapham, Caroline \& Wall, Diane (1995[1998]). Exámenes de idiomas. Elaboración y evaluación. Madrid: Cambridge University Press.

Alonso Belmonte, Isabel (2004). «La subcompetencia discursiva». En J. Sánchez Lobato e I. Santos Gargallo (dir.); Vademécum para la formación de profesores. Enseñar español como segunda lengua (L2)/Lengua extranjera (LE) (pp. 553-572). Madrid: SGEL.

Álvarez Angulo, Teodoro (2005). Didáctica del texto en la formación del profesorado. Madrid: Síntesis.

Andon, Nick \& Wingate, Ursula (2013). «Motivation, Authenticity and Challenge in German Textbooks for Key Stage 3». En J. Gray (ed.); Critical Perspectives on Language Teaching Materials (pp. 182-203). Basingstoke: Palgrave MacMillan.

BroncKart, Jean-Paul (2007). Desarrollo del lenguaje y didáctica de las lenguas. Buenos Aires: Miño y Dávila.

Burns, Anne (2012). «Text-Based Teaching». En A. Burns y J.C. Richards (ed.); The Cambridge Guide to Pedagogy and Practice in Second Language Teaching (pp. 140148). Cambridge: Cambridge University Press.

Celce-Murcia, Marianne, DörnYeI, Zoltán \& Thurrell, Sarah (1995). «Communicative Competence: A pedagogically motivated model with content specifications». Issues in Applied Linguistics, 6, 5-35.

CuEnCA, Maria Josep (2008). Gramàtica del text. Alzira: Bromera.

DUQue, Eladio (2016). Las relaciones de discurso. Madrid: Arco Libros.

FEEZ, Susan \& JOYCE, Helen (1998). Text-based syllabus design. Sídney: NCELTR.

FERrer, Montserrat y RodríGuez, Carmen (2010). «La lingüística del texto en los materiales de la ESO». En T. Ribas Seix (coord.); Libros de texto y enseñanza de la gramática (pp. 97-116). Barcelona: Graó.

FuENTES RodRíGUEZ, Catalina (2000). Lingüistica pragmática y análisis del discurso. Madrid: Arco Libros. 
HASSON, Uri \& SMALL, Steven L. (2008). «Functional Magnetic Resonance Imaging (fMRI) Research of Language». En B. Stemmer y H.A. Whitaker (ed.); Handbook of the neuroscience of language (pp. 81-89). Ámsterdam: Academic Press/Elsevier.

HYMES, Dell H. (1971 [1995]). «Acerca de la competencia comunicativa». En M. Llobera (comp.); Competencia comunicativa. Documentos básicos en la enseñanza de lenguas extranjeras (pp. 27-46). Madrid: Edelsa.

Instituto Cervantes (2006). Plan curricular del Instituto Cervantes. Niveles de referencia para el español. Madrid: Biblioteca Nueva.

Maley, Alan \& Prowse, Philip (2013). «Reading». En B. Tomlinson (ed.); Applied Linguistics and Materials Development (pp. 165-182). Londres: Bloomsbury.

MARTÍN PERIS, Ernesto (dir.) (2008). Diccionario de términos clave de ELE. Madrid: SGEL.

MASUHARA, Hitomi (2016). «Brain Studies and Materials for Language Learning». En B. Tomlinson (ed.); SLA Research and Materials Development for Language Learning, (pp. 23-32). Nueva York: Routledge.

MCCARTHY, Michael \& CARTER, Ronald (1994). Language as Discourse. Perspectives for Language Teaching. Harlow: Longman.

MCGRATH, Ian (2016). Materials Evaluation and Design for Language Teaching. Second Edition. Edimburgo: Edinburgh University Press.

MORENO CABRERA, Juan Carlos (2002). Curso universitario de lingüística general 1: Teoría de la gramática y sintaxis general, Madrid, Síntesis.

MuÑoz PÉREZ, Julián (2017). Sobre el tratamiento de la subcompetencia discursivotextual en los manuales de español como lengua extranjera: análisis de corpus y propuestas didácticas de mejora. Tesis doctoral inédita.

Pagola, José Antonio (2011). «Fidelidad a Dios y a la Tierra». En J.A. Pagola; El camino abierto por Jesús 2. Marcos (pp. 161-162). Bilbao: Desclée de Brower.

RiggenBACH, Heidi. (1999). Discourse Analysis in the Language Classroom. Volume 1. The Spoken Language. Ann Arbor: The University of Michigan Press.

SERRANO, María José (2006). Gramática del discurso. Madrid: Akal.

THORNBURY, Scott (2005). Beyond the sentence. Introducing discourse analysis. Oxford: Macmillan.

Tomlinson, Brian (ed.) (2013). Applied Linguistics and Materials Development. Londres: Bloomsbury.

VAN BERKUM, Jos J.A. (2012). «The Electrophysiology of Discourse and Conversation». En M.J. Spivey, K. McRae y M.F. Joanisse (eds.); The Cambridge Handbook of Psycholinguistics (pp. 589-612). Cambridge: Cambridge University Press.

Wennerstrom, Ann (2003). Discourse Analysis in the Language Classroom. Volume 2. Genres of Writing. Ann Arbor: The University of Michigan Press. 